\title{
Long noncoding RNA CRNDE promotes proliferation, migration and invasion in prostate cancer through miR-101/Rap1A
}

\author{
J. H. CHEN, W. TONG, X.F. PU, J. Z. WANG* \\ Department of Urology Surgery, The Chongqing General Hospital, Yuzhong, Chongqing, China \\ ${ }^{*}$ Correspondence: $y z x b j b @ 163 . c o m$
}

Received June 21, 2019 / Accepted October 2, 2019

\begin{abstract}
Prostate cancer (Pca) is the second frequent malignancy in men. Long noncoding RNAs (lncRNAs) have been reported to play essential roles in the progression of cancers, including Pca. LncRNA colorectal neoplasia differentially expressed (CRNDE) has been found to affect tumorigenesis in many cancers. However, the exact role and mechanism of CRNDE in Pca remain poorly understood. 64 Pca patients were recruited in this study. PC3 and 22RV1 cells were used in vitro experiments. The expressions of CRNDE, microRNA-101 (miR-101), and Ras-related protein 1A (Rap1A) were detected in vivo and in vitro by quantitative real-time polymerase chain reaction and western blot, respectively. Cell proliferation, apoptosis, migration, and invasion were investigated through cell counting kit-8, flow cytometry, and Transwell assays, respectively. The interaction between miR-101 and CRNDE or Rap1A was explored by bioinformatics analysis and luciferase reporter assay. High expression of CRNDE was shown in Pca tissues and cells and predicted poor outcomes of patients. Overexpression of CRNDE promoted cell proliferation, migration, and invasion but decreased apoptosis in Pca cells, while its knockdown showed an opposite effect. CRNDE was a decoy of miR-101 and its effect on Pca progression was reversed by miR-101. Rap1A was identified as a target of miR-101 and it attenuated the effect of miR-101 on Pca development. Moreover, the Rap1A protein level was positively regulated by CRNDE, which was weakened by miR-101. CRNDE contributed to cell proliferation, migration, and invasion by regulating the miR-101/Rap1A axis in Pca, providing a novel strategy for Pca treatment.
\end{abstract}

Key words: prostate cancer, CRNDE, miR-101, Rap1A

Prostate cancer (Pca) is one of the most common malignancies with a major cause of cancer deaths in men worldwide [1]. Surgery is the main curative treatment with an adverse effect on life quality, such as urinary symptoms and sexual dysfunction. In recent years, much advance has been made in the diagnosis and treatment of Pca, but the effective strategies remain limited [2]. Hence, it is urgent to explore new avenues for therapeutics for patients with Pca.

Noncoding RNAs (ncRNAs), including long noncoding RNAs (lncRNAs) and microRNAs (miRNAs), play important roles in pathogenesis, progression, diagnosis, and treatment of endocrine-related cancers [3]. There is a competitive regulatory interaction in ncRNAs, thus $\operatorname{lncRNA}$ could act as a competing endogenous RNA (ceRNA) for miRNA to regulate gene expression [4]. LncRNAs longer than 200 nucleotides without the capacity of coding proteins are implicated in cancer progression and metastasis [5]. Moreover, lncRNAs are reported as vital targets for the diagnosis and treatment of Pca [6]. LncRNA colorectal neoplasia differentially expressed
(CRNDE) has been demonstrated as an important oncogene to promote the progression of cancers, such as oral squamous cell carcinoma, non-small cell lung cancer, and hepatocellular carcinoma [7-9]. However, little is known about the biological role of CRNDE in Pca.

miRNAs targeting mRNAs to reduce their expression participate in cancer progression and play essential roles in diagnosis, prognosis, and management of Pca [10]. The previous study revealed that miR-101 could serve in cancer therapy as a tumor suppressor by regulating cell processes, such as proliferation, apoptosis, metastasis, and drug resistance [11]. More importantly, it was reported that miR-101 is lowly expressed in Pca [12]. Furthermore, increasing works suggested the potential therapeutic effect of miR-101 in Pca $[13,14]$. Ras-related protein $1 \mathrm{~A}$ (Rap1A) is an oncogene, which plays an essential role in the progression of cancers, such as cervical cancer, breast cancer, and ovarian cancer [15-17]. In addition, a former effort reported Rap1A as a target of miR-203 to regulate cell proliferation, adhesion, and 
invasion in Pca [18]. Based on the binding sites of miR-101 and CRNDE or Rap1A predicted by bioinformatics analysis, we hypothesized that miR-101 and Rap1A might be associated with CRNDE-mediated Pca progression. In this study, we found that CRNDE expression was enhanced in Pca. Moreover, using PC3 and 22RV1 cells, we investigated the effect of CRNDE on Pca proliferation, migration, and invasion in vitro. Besides, we explored the ceRNA regulatory network of CRNDE/miR-101/Rap1A.

\section{Patients and methods}

Patients and tissues. A total of 64 patients with Pca were recruited from the Chongqing General Hospital and they have provided the informed consent. The cancer specimens and adjacent para-tumor tissues were harvested from the same participant and stored at $-80^{\circ} \mathrm{C}$ for RNA extraction. The procedures in this research were approved by the ethics committee of the Chongqing General Hospital. The overall survival of patients was analyzed by follow-up on a monthly basis. The clinicopathological characteristics of the patients were summarized in Table 1.

Cell culture and transfection. The normal prostate stromal cell (WPMY-1) and human Pca cell lines (PC-3, DU-145, LnCap, and 22RV1) were purchased from the Shanghai Institute of Cell Bank (Shanghai, China). RPMI-1640 medium with $10 \%$ fetal bovine serum was used for cell culture at $37^{\circ} \mathrm{C}$ under $5 \% \mathrm{CO}_{2}$. Small interfering RNA (siRNA) against CRNDE (si-CRNDE\#1, \#2, \#3), siRNA against Rap1A (si-Rap1A), siRNA negative control (si-NC), pcDNA3.1based CRNDE overexpression vector (pcDNA3.1-CRNDE), pcDNA3.1-based Rap1A overexpression vector (pcDNA3.1Rap1A), pcDNA3.1 empty vector (pcDNA3.1-NC), miR-101 mimics, miRNA negative control (miR-NC mimics), miR-101 inhibitor and inhibitor negative control (miR-NC inhibitor) were obtained from Genepharma (Shanghai, China) and transfected into Pca cells using LipoFiter ${ }^{\mathrm{TM}}$ Liposomal Trans- $^{-}$ fection Reagent (Hanbio, Shanghai, China) following the manufactures' instructions. After $24 \mathrm{~h}$, transfected cells were harvested for further analyses.

Quantitative real-time polymerase chain reaction (qRT-PCR). RNA was isolated using Trizol (Thermo Fisher, Waltham, MA, USA) following the manufacturer's protocols and analyzed with a NanoDrop ND-2000 spectrophotometer (Thermo Fisher). The samples with optical density (OD) 260/280 between 1.8-2.0 were selected for reverse transcription using a first strand cDNA kit (Sigma, St. Louis, MO, USA). The qRT-PCR was performed using SYBR Green (TaKaRA, Dalian, China) and a sample of each group was prepared in duplicate. The primers used in this study were listed as follows: CRNDE (Forward, 5'-CGCGCCCGCGCGGCGGAGGA-3'; Reverse, 5'-TATGAATTGCAGACTTTGCAGA-3'); Rap1A (Forward, 5'-CGTGAGTACAAGCTAGTGGTCC-3'; Reverse, 5'-CCAGGATTTCGAGCATACACTG-3'); GAPDH (Forward, 5'-GTCAACGGATTTGGTCTG-
TATT-3'; Reverse, 5'-AGTCTTCTGGGTGGCAGTGAT-3'); miR-101 (Forward, 5'-CGGCGGTACAGTACTGTGATAA-3'; Reverse, 5'-CTGGTGTCGTGGAGTCGGCAATTC-3'); U6 (Forward, 5'-CTTCGGCAGCACATATAC-3'; Reverse, 5'-TTCACGAATTTGCGTGTCAT-3').

The relative expression levels of CRNDE, miR-101, and Rap1A were normalized to its control and calculated according to the $2^{-\Delta \Delta \mathrm{Ct}}$ method [19].

Cell proliferation assay. Cell proliferation was examined by using cell counting kit-8 (CCK-8) (Beyotime, Shanghai, China). Transfected PC3 and 22RV1 cells were seeded into 96-well plates at a density of $3 \times 10^{3}$ cells/well in triplicate. At $1,2,3$ and 4 day, cells were incubated with CCK- 8 reagent for $2 \mathrm{~h}$. Subsequently, the OD of each well at $450 \mathrm{~nm}$ was determined using a microplate reader (Bio-Rad, Hercules, CA, USA).

Cell apoptosis assay. Cell apoptosis was detected with an Annexin V-FITC/PI apoptosis detection kit (Yeasen, Shanghai, China) through flow cytometry. Transfected PC3 and $22 \mathrm{RV} 1$ cells $\left(5 \times 10^{4}\right)$ were seeded into 6 -well plates and cultured for 4 days. After digested and washed, cells were collected and incubated with $5 \mu \mathrm{l}$ Annexin V-FITC and PI in the dark for $5 \mathrm{~min}$. The determination of the apoptotic rate was performed using a flow cytometer (Becton Dickinson, San Jose, CA, USA).

Transwell assay. Transwell assay was used for the analysis of cell migration and invasion using 24-well transwell chambers (Corning, Corning, NY, USA). The chamber was pre-coated with Matrigel (Becton Dickinson) with respect to the invasion assay. Briefly, PC 3 and $22 \mathrm{RV} 1$ cells $\left(1 \times 10^{4}\right.$ cells in serum-free medium) were seeded into upper chambers

Table 1. The clinicopathological characteristics of prostate patients.

\begin{tabular}{|c|c|c|c|c|}
\hline \multirow[b]{2}{*}{ Parameters } & \multirow[b]{2}{*}{ Total } & \multicolumn{2}{|c|}{ CRNDE expression } & \multirow[b]{2}{*}{ p-value } \\
\hline & & $\begin{array}{c}\text { High } \\
(n=36)\end{array}$ & $\begin{array}{c}\text { Low } \\
(n=28)\end{array}$ & \\
\hline \multicolumn{5}{|l|}{ Age } \\
\hline$<65$ & 18 & 8 & 10 & 0.430 \\
\hline$\geq 65$ & 46 & 28 & 18 & \\
\hline \multicolumn{5}{|c|}{ Tumor diameter } \\
\hline$<2.0$ & 25 & 9 & 16 & $0.008^{*}$ \\
\hline$\geq 2.0$ & 39 & 27 & 12 & \\
\hline \multicolumn{5}{|c|}{ Gleason score } \\
\hline$<7$ & 30 & 22 & 18 & 0.247 \\
\hline$\geq 7$ & 34 & 14 & 20 & \\
\hline \multicolumn{5}{|l|}{ Tumor stage } \\
\hline $\mathrm{T} 2$ & 28 & 10 & 18 & $0.023^{*}$ \\
\hline T3-T4 & 36 & 26 & 10 & \\
\hline \multicolumn{5}{|c|}{ Lymph node metastasis } \\
\hline No & 38 & 16 & 22 & $0.004^{*}$ \\
\hline Yes & 26 & 20 & 6 & \\
\hline \multicolumn{5}{|c|}{ Distant metastasis } \\
\hline No & 30 & 13 & 17 & $0.011^{\star}$ \\
\hline Yes & 34 & 23 & 11 & \\
\hline
\end{tabular}


and the lower chamber was filled with $500 \mu \mathrm{l}$ medium with $10 \%$ fetal bovine serum. After culturing for $24 \mathrm{~h}$ at $37^{\circ} \mathrm{C}$, cells penetrating the membrane were stained with $0.1 \%$ crystal violet (Sigma) for $3 \mathrm{~min}$. The stained cells were observed under an inverted microscope $(\times 200$ magnification, Olympus, Tokyo, Japan) with three randomly selected fields.

Bioinformatics analysis and luciferase reporter assay. Bioinformatics analysis using Microcode and TargetScan provided the predicted binding sites of miR-101 and CRNDE or Rap1A. Luciferase assay was performed in PC3 cells. Wild type (WT) sequences of CRNDE containing the predicted binding sites of miR-101 (UACUGUA) were amplified and cloned into firefly luciferase-expressing pmirGLO vectors (Promega, Madison, WI, USA) to generate CRNDE-WT. Its mutant (MUT) was generated by changing the seed sites to AGUCUCC, named as CRNDE-MUT. The WT or MUT 3' UTR sequences of Rap1A mutating miR-101 binding sites (UACUGUA) to CGAAUCC were also used for the establishment of luciferase reporter vectors, named as Rap1A-WT or Rap1A-MUT, respectively. The cells were co-transfected with WT or MUT luciferase reporter constructs and miR-101 mimics or miR-NC mimics, along with renilla vector at the same time using LipoFiter ${ }^{\text {tw }}$ Liposomal Transfection Reagent. Cells were harvested after $48 \mathrm{~h}$ and luciferase activity was analyzed using a luciferase reporter assay kit (Promega).

Western blot. After the transfection, PC3 and 22RV1 cells were collected and lysed in RIPA buffer with 1\% PMSF (Yeasen), followed by thehigh-speed centrifugation $(12000 \times \mathrm{g}$, $10 \mathrm{~min})$. The protein in the supernatant was quantified using Enhanced BCA Protein Assay Kit (Beyotime) and denatured in Sample Loading Buffer (Beyotime) at $98^{\circ} \mathrm{C}$ for $10 \mathrm{~min}$. Samples were prepared three times. Proteins were loaded onto the SDS-PAGE gel and the nitrocellulose membranes (Millipore, Billerica, MA, USA) were used to transfer the proteins with western transfer buffer (Beyotime), and the nonspecific binding sites of membranes were blocked using QuickBlock $^{\text {Tm }}$ Blocking Buffer (Beyotime). The anti-Rap1A (ab96223) and anti-GAPDH (ab181602) primary antibodies and corresponding secondary antibody (ab6721) used in this study were purchased from Abcam (Cambridge, MA, USA).
The BeyoECL Plus (Beyotime) was used for visualization of protein signals. The expression level of Rap1A was expressed as Rap1A/GAPDH ratio according to the densitometry analysis using Image Lab software (Bio-Rad).

Statistical analysis. Statistical analysis and results display were conducted by GraphPad Prism 7 software (GraphPad Inc., La Jolla, CA, USA). The experiments were repeated three times and data of each group was expressed as mean \pm standard deviation (S.D.). The overall survival curve of Pca patients was generated by the Kaplan-Meier method and analyzed by the Log-rank test. The linear correlations among CRNDE, miR-101 and Rap1A expression level in Pca tissues were investigated by Spearman's correlation analysis. The difference between groups was analyzed by Student's t-test or ANOVA and it was significant when $\mathrm{p}<0.05$.

\section{Results}

CRNDE expression is enhanced in Pca. To explore the role of CRNDE in Pca, its expression was measured in Pca. Compared with that in adjacent samples, the expression of CRNDE was aberrantly enhanced in Pca tissues $(\mathrm{n}=64$, Figure 1A). Moreover, the level of CRNDE was significantly elevated in Pca cells (PC3, DU145, LnCap, and 22RV1) compared with that in WPMY-1 cells (Figure 1B). In addition, by dividing the patients as high $(\mathrm{n}=36)$ or low CRNDE $(n=28)$ expression group according to its mean value, the high CRNDE expression was associated with poor overall survival, tumor diameter, tumor stage, lymph node metastasis, and distant metastasis $(\mathrm{p}<0.05)$ (Figure 1C, Table 1). These findings suggested that the high expression of CRNDE predicted poor outcomes of patients. Meanwhile, according to the CRNDE level in the four Pca cell lines, PC3 cells with a relative higher CRNDE level and 22RV1 cells with a relative lower level were selected for further in vitro experiments.

CRNDE promotes cell proliferation, migration, and invasion in Pca cells. To investigate the effect of CRNDE on Pca progression, PC3 cells were transfected with si-NC or three si-CRNDE and 22RV1 cells were transfected with
A

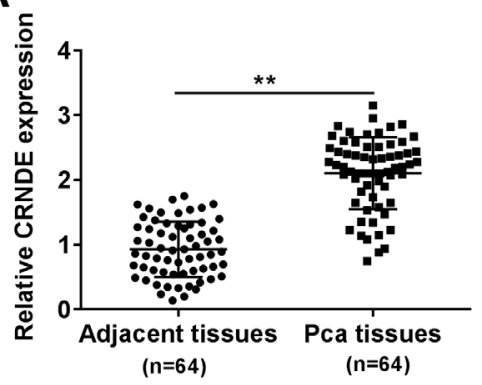

B

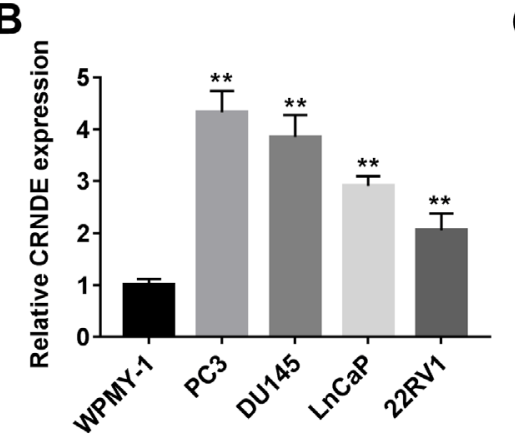

C

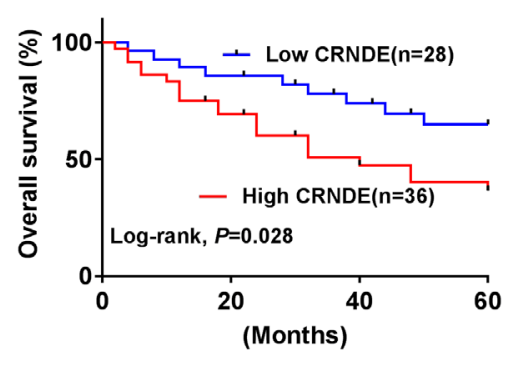

Figure 1. The expression of CRNDE is enhanced in Pca. A) The expression of CRNDE was measured in Pca tissues and adjacent samples by qRT-PCR, $\mathrm{n}=64$. B) The level of CRNDE was detected in Pca cells and normal prostate stromal cells (WPMY-1) by qRT-PCR. C) The overall survival of patients was analyzed according to the mean value of the CRND1 level in Pca tissues, ${ }^{* *} \mathbf{p}<0.01$. 
A

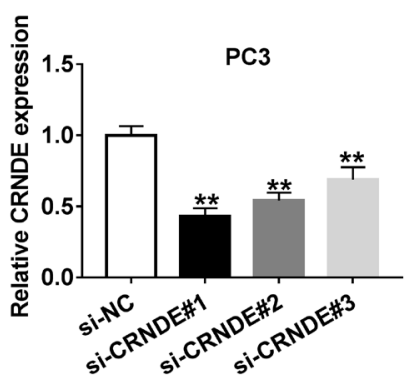

D

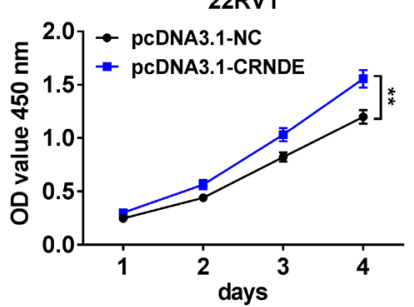

B

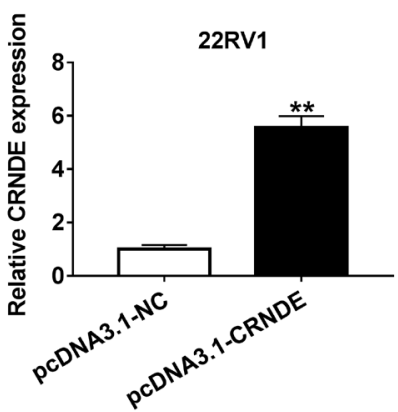

E

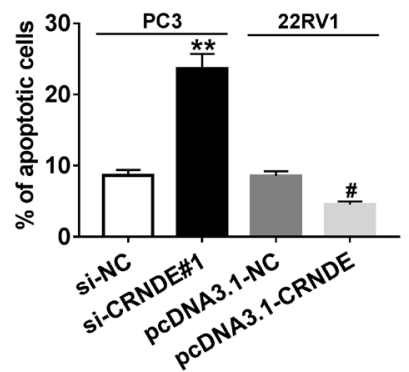

C

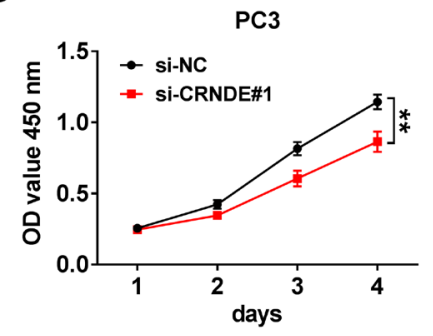

$\mathbf{F}$

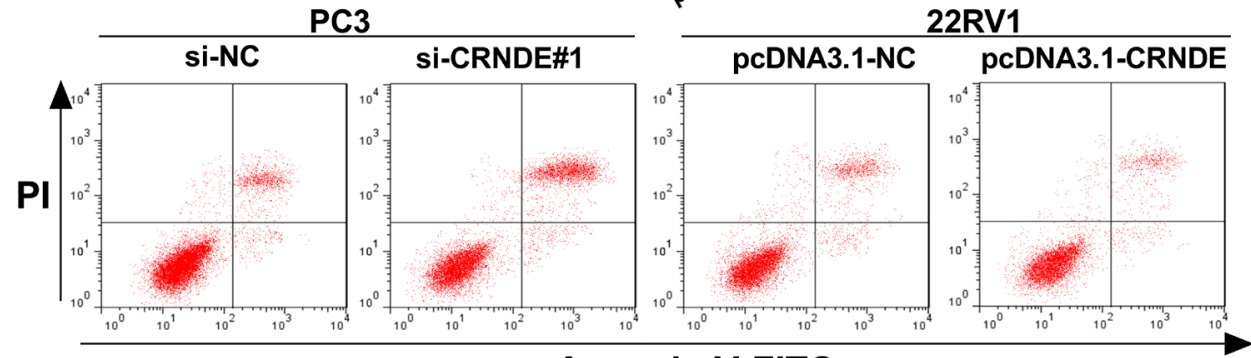

Annexin V-FITC

G

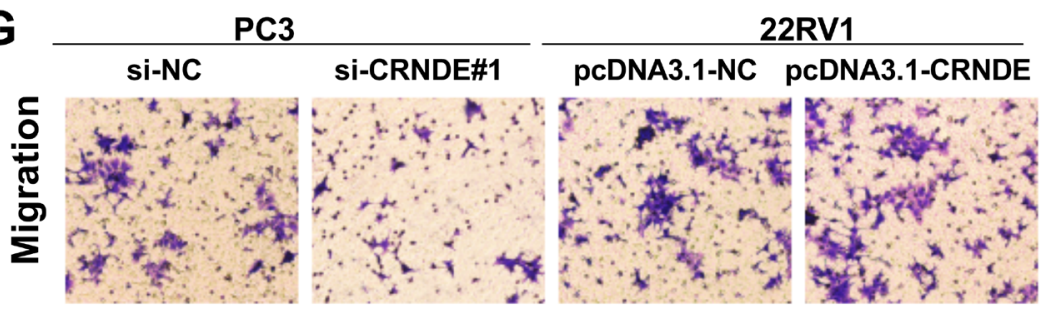

H

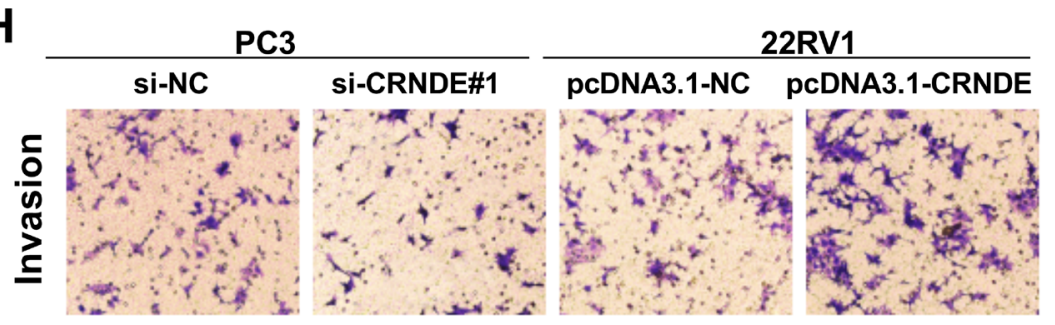

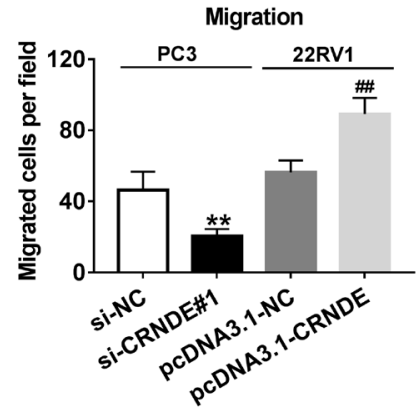

Migration

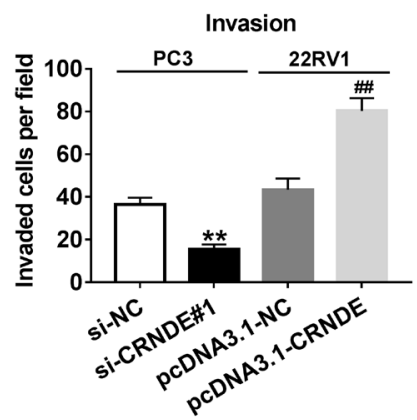

Figure 2. CRND1 regulates Pca progression in vitro. PC3 cells were transfected with si-NC or 3 si-CRNDE and 22RV1 cells were transfected with pcDNA3.1-NC or pcDNA3.1-CRNDE. The expression of CRNDE (A, B), cell proliferation $(C, D)$, apoptosis $(E$, F), migration and invasion $(G, H)$ were measured in the two transfected cell lines by qRT-PCR, CCK-8, flow cytometry, and transwell assays, respectively, ${ }^{* *} \mathrm{p}<0.01,{ }^{*} \mathrm{p}<0.05,{ }^{* *} \mathrm{p}<0.01$. 
pcDNA3.1-NC or pcDNA3.1-CRNDE. The transfection efficacy was validated by qRT-PCR with the results of reduced CRNDE level in PC3 and increased expression in 22RV1 cells in comparison to their corresponding controls (Figures 2A, 2B). Subsequently, the cell progression was investigated. As shown in Figures $2 \mathrm{C}$ and $2 \mathrm{D}, \mathrm{CCK}-8$ assay revealed that knockdown of CRNDE significantly decreased cell proliferation in PC3 after a culture of 4 days, while its overexpression promoted 22RV1 cell proliferation. Moreover, analysis of flow cytometry demonstrated that the apoptotic rate was notably increased in PC3 cells by silencing CRNDE but inhibited by its overexpression in 22RV1 cells (Figures 2E, $2 \mathrm{~F})$. In addition, cell migration and invasion were analyzed by the transwell assay after $24 \mathrm{~h}$ when the cell proliferation was not significantly affected in each group. As described in Figures $2 \mathrm{G}$ and $2 \mathrm{H}$, results showed that interference of CRNDE evidently suppressed the abilities of migration and invasion, but its overexpression played an opposite effect. These results showed that CRNDE promoted Pca progression.

CRNDE regulates Pca progression by sponging miR-101. miRNAs are the key biomarkers involved in the functional lncRNAs. To explore the underlying mechanism by which CRNDE regulated Pca progression, bioinformatics analysis was performed using Microcode and provided the binding sites of CRNDE and miR-101 (Figure 3A), suggesting the potential interaction between them. To identify this prediction, luciferase reporter assay using CRNDE-WT and CRNDE-MUT was performed in PC3 cells. As displayed in Figure $3 \mathrm{~B}$, the overexpression of miR-101 markedly reduced the luciferase activity in the CRNDE group, whereas its efficacy was lost in the CRNDE-MUT group. Moreover, the expression of miR-101 was measured in Pca tissues and results showed that the miR-101 expression was significantly decreased in Pca tissues compared with that in adjacent
A

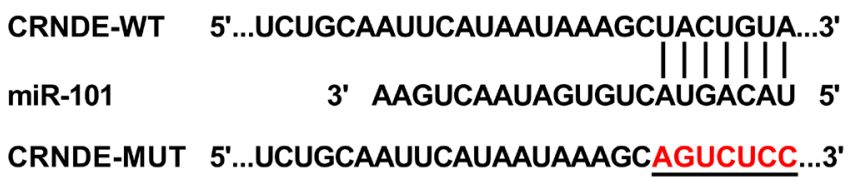

C

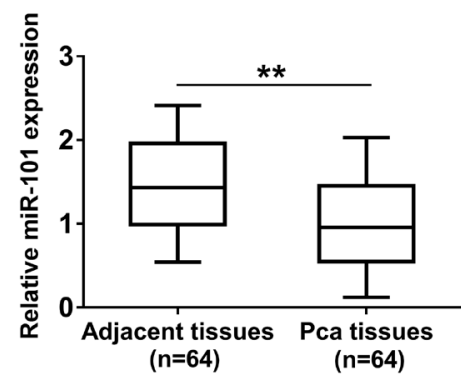

$\mathbf{F}$

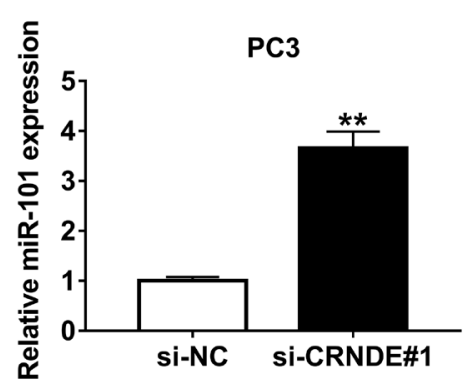

G

22RV1
B

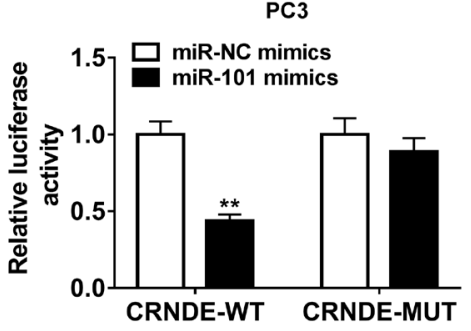

E

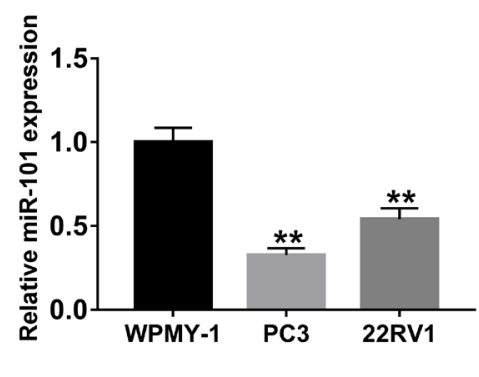

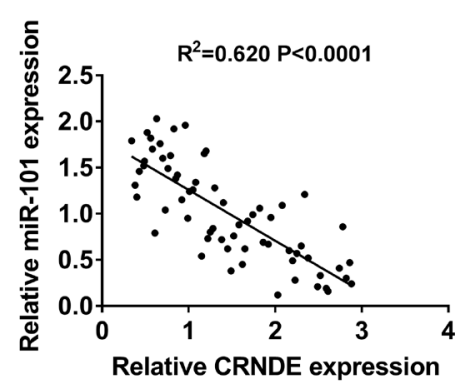

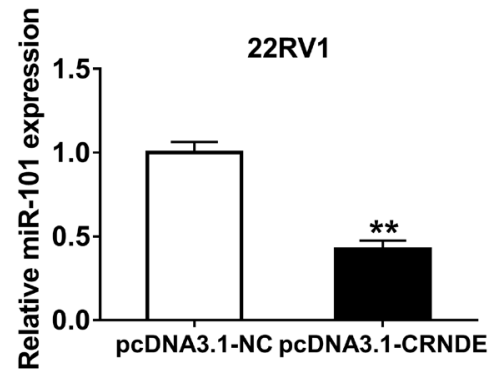

Figure 3. CRNDE is a sponge of miR-101. A) The binding sites of CRNDE and miR-101 were predicted by Microcode. B) Luciferase activity was measured in PC3 cells co-transfected with miR-101 mimics or miR-NC mimics and CRNDE-WT or CRNDE-MUT. C) The expression of miR-101 was measured in Pca tissues by $q R T-P C R, n=64$. D) The relationship between CRNDE and miR-101 level in Pca tissues was analyzed. E) The level of miR-101 was detected in Pca cells by qRT-PCR. F, G) The expression of miR-101 was measured in PC3 cells transfected with si-NC or si-CRNDE and 22RV1 cells transfected with cDNA3.1-NC or pcDNA3.1-CRNDE, ${ }^{* *} \mathrm{p}<0.01$. 
A

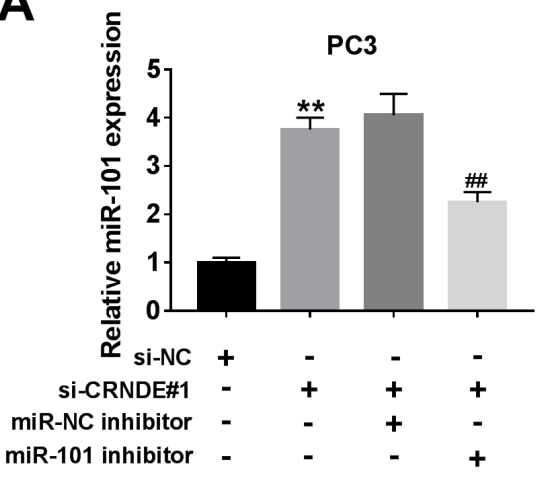

D

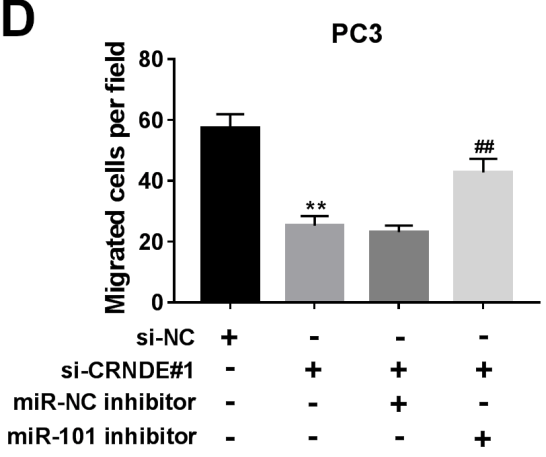

F

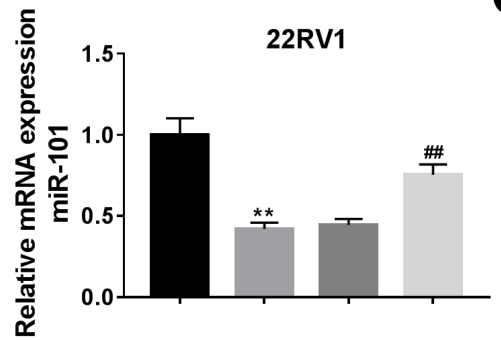

PCDNA3.1-NC +

pcDNA3.1-CRNDE
miR-NC mimics miR-101 mimics
B

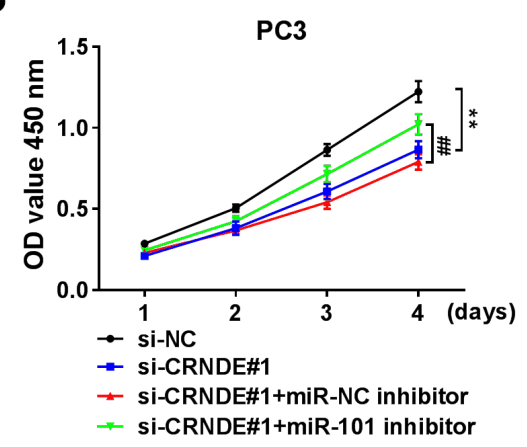

E

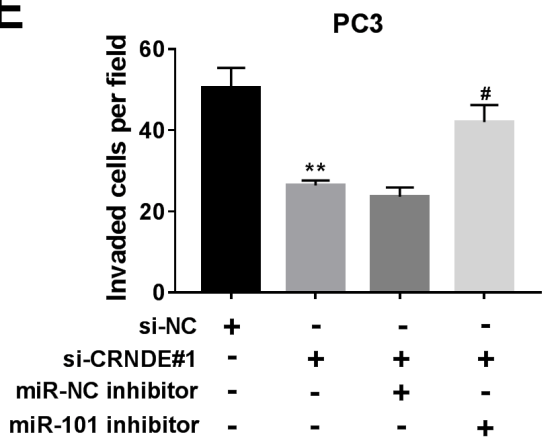

C $\mathrm{PC} 3$

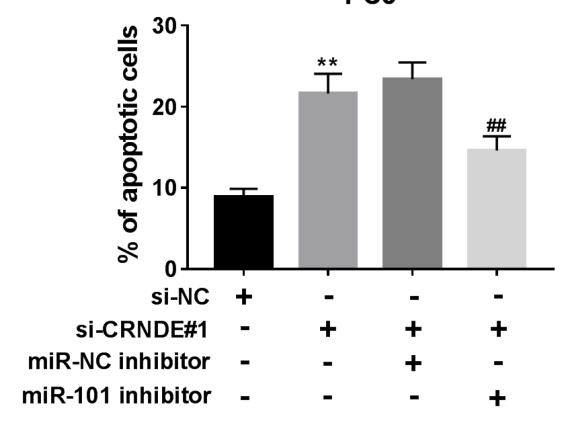

G

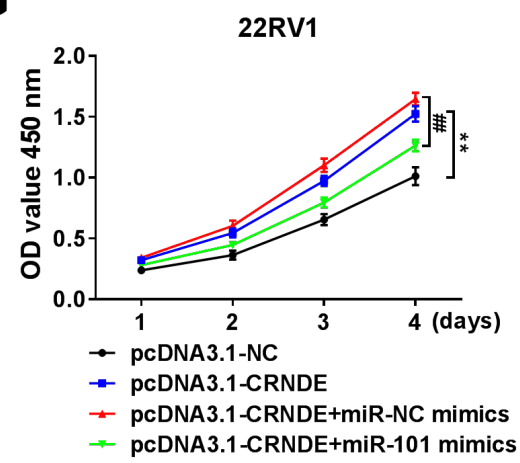

H

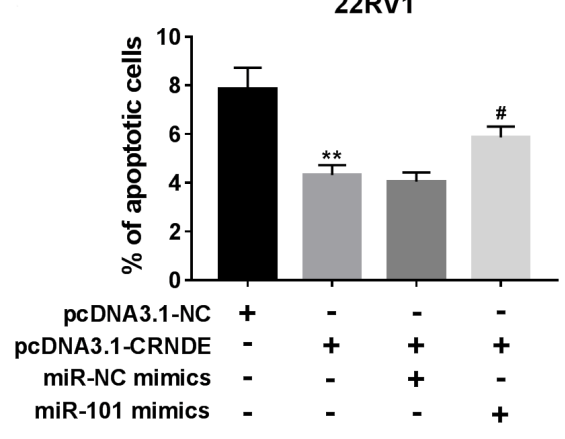

I

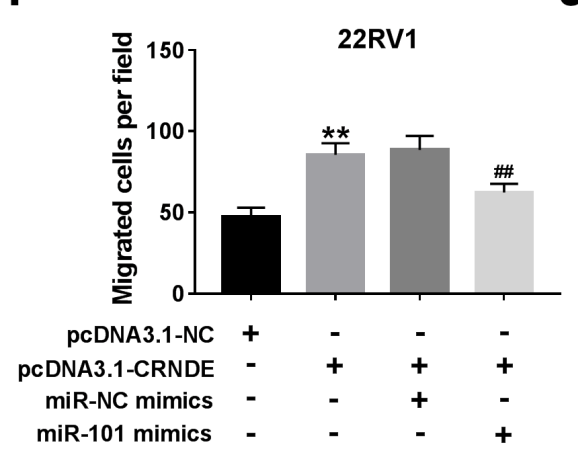

J

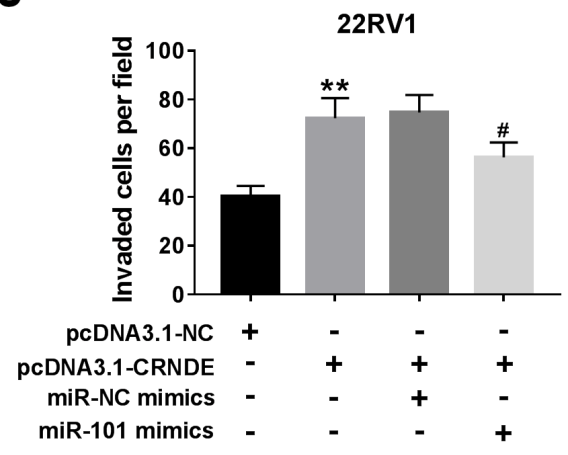

Figure 4. miR-101 reverses the effect of CRNDE on Pca progression. PC3 cells were transfected with si-NC, si-CRNDE, si-CRNDE, and miR-NC inhibitor or miR-101 inhibitor. The expression of miR-101 (A), cell proliferation (B), apoptosis (C), migration, and invasion (D, E) were measured in transfected PC3 cells by qRT-PCR, CCK-8, flow cytometry, and transwell assays, respectively. 22RV1 cells were transfected with pcDNA3.1-NC, pcDNA3.1-CRNDE, pcDNA3.1-CRNDE, and miR-101 mimics or miR-NC mimics. The expression of miR-101 (F), cell proliferation (G), apoptosis (H), migration, and invasion (I, J) were detected in transfected $22 \mathrm{RV} 1$ cells, ${ }^{* *} \mathrm{p}<0.01,{ }^{*} \mathrm{p}<0.05,{ }^{* * *} \mathrm{p}<0.01$. 
groups (Figure 3C). Meanwhile, its expression was negatively associated with CRNDE level in patients' tissues $\left(R^{2}=0.620\right.$, $\mathrm{p}<0.000$, Figure 3D). Similarly, comparing with WPMY-1 cells, PC3 and 22RV1 cells exhibited lower expression of miR-101 (Figure 3E). In addition, according to the data of qRT-PCR, the expression of miR-101 was greatly enhanced by the CRNDE silence but decreased by the CRNDE overexpression (Figures 3F, 3G). These findings indicated CRNDE as a decoy of miR-101.

To explore whether the effect of CRNDE on Pca progression was modulated by miR-101, the rescue experiments were conducted in the two cell lines. In PC3 cells by transfection of si-NC, si-CRNDE, si-CRNDE and miR-NC inhibitor or
miR-101 inhibitor, the expression of miR-101 elevated by the CRNDE knockdown was significantly downregulated via the transfection of the miR-101 inhibitor (Figure 4A). Moreover, the cell progression assay showed that deficiency of miR-101 attenuated the knockdown of CRNDE-mediated inhibition of proliferation, migration and invasion, and promotion of apoptosis in PC3 cells (Figures 4B-E). Furthermore, rescue experiments were performed in 22RV1 cells and the miR-101 level was restored by the introduction of miR-101 mimics (Figure 4F). Besides, the upregulation of miR-101 weakened the promoting role of CRNDE in Pca progression (Figures 4G-J). These data uncovered that CRNDE inhibited miR-101 to promote Pca development.
A

Position 1029-1035 of Rap1A

Rap1A-WT 5'...AAAAGAUAAGUUUAAUACUGUAU...3'

miR-101

3' AagucaAuagugucaugacAu 5'

Rap1A-MUT 5'...AAAAGAUAAGUUUAACGAAUCCU....3'
B

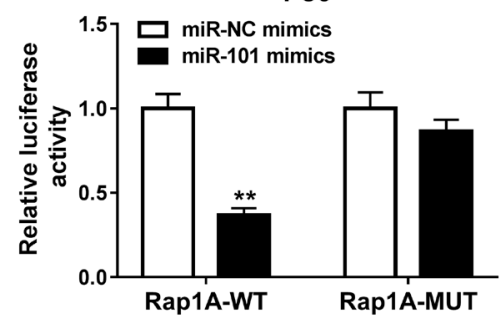

C

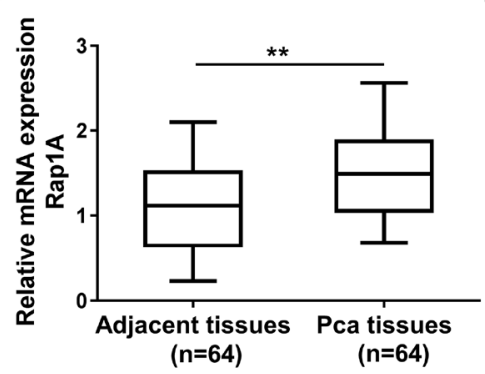

$\mathbf{F}$

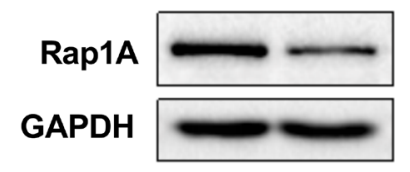

$\mathrm{PC} 3$

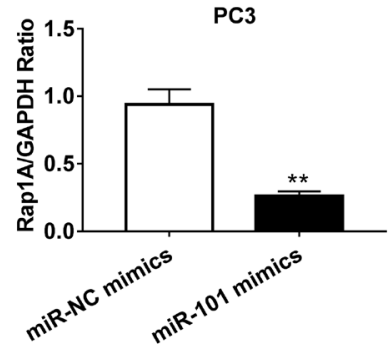

D

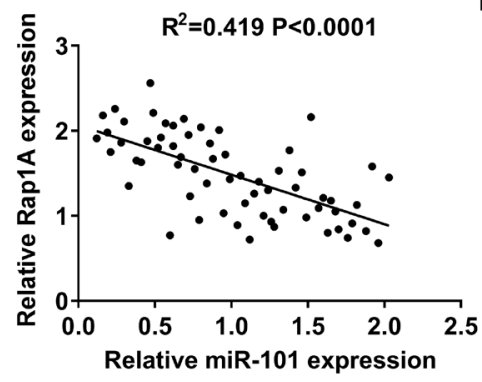

E

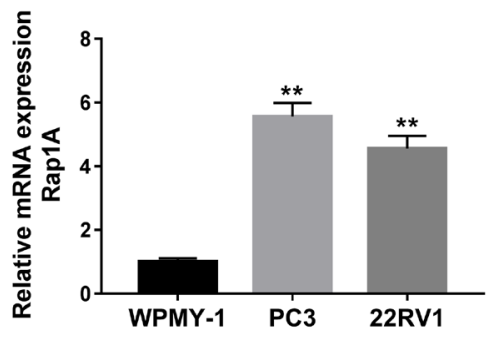

G

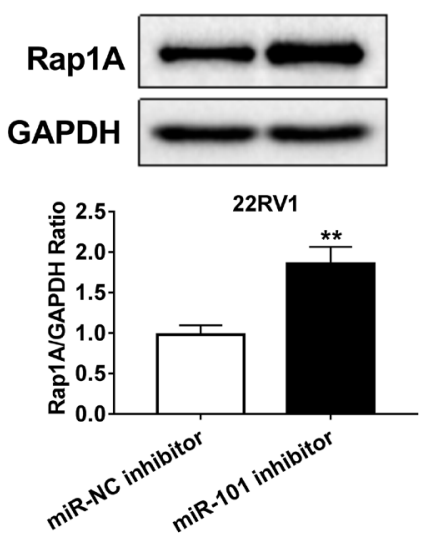

Figure 5. Rap1 A is a target of miR-101. A) The binding sites of miR-101 and Rap1A were provided by TargetScan. B) Luciferase activity was measured in PC3 cells co-transfected with miR-101 mimics or miR-NC mimics and Rap1A-WT or Rap1A-MUT. C) The expression of Rap1A mRNA was measured in Pca tissues by qRT-PCR, $n=64$. D) The association between Rap1 A and miR-101 level in Pca tissues was analyzed. E) The mRNA level of Rap1A was detected in Pca cells by qRT-PCR. F, G) The expression of Rap1 A protein was measured in PC3 cells transfected with miR-101 mimics or miR-NC mimics and 22RV1 cells transfected with miR-101 inhibitor or miR-NC inhibitor by western blot, ${ }^{* *} \mathrm{p}<0.01$. 
miR-101 inhibits Pca progression by targeting Rap1A. Targeted genes are required for the functional miRNAs. As shown in Figure 5A, bioinformatics analysis using TargetScan predicted the binding sites of miR-101 and Rap1A. This association was proved by the luciferase reporter assay with the results that the overexpression of miR-101 reduced the luciferase activity in PC3 cells transfected with Rap1A-WT but not in Rap1A-MUT-transfected cells (Figure 5B), suggesting that Rap1A could be a target of miR-101. Subsequently, the expression of Rap1A was measured in Pca tissues and cells. The expression of Rap1A mRNA was significantly upregulated in Pca tissues $(n=64)$ and cells, and negatively correlated with miR-101 level $\left(R^{2}=0.419, p<0.0001\right.$, Figures $\left.5 C-E\right)$. Moreover, the Rap1A protein level was decreased by the miR-101 overexpression and increased by the miR-101 inhibition in Pca cells (Figures 5F, 5G). These results displayed that miR-101 could target and decrease the Rap1A expression.

To explore whether Rap1A was involved in the miR-101mediated Pca progression, PC3 cells were transfected with miR-NC mimics, miR-101 mimics, miR-101 mimics, and pcDNA3.1-NC or pcDNA3.1-Rap1A and 22RV1 cells were transfected with miR-NC inhibitor, miR-101 inhibitor, miR-101 inhibitor, and si-NC or si-Rap1A. After the transfection, the expression levels of Rap1A mRNA and protein were obviously inhibited in PC3 cells by overexpression of miR-101, while it was reversed by the introduction of the Rap1A overexpression vector (Figures 6A, 6B). The further functional analyses revealed that the miR-101 overexpression inhibited cell proliferation, migration, and invasion but promoted apoptosis in PC3 cells, which were counteracted by the restoration of Rap1A (Figures 6C-F). However, in $22 \mathrm{RV} 1$ cells, the Rap1A expression at mRNA and protein levels was increased by the miR-101 knockdown, which was weakened by silencing Rap1A (Figures 6G, 6H). Meanwhile, the miR-101 exhaustion significantly promoted Pca progression, which was alleviated by silencing Rap1A in 22RV1 cells (Figures 6I-L). These results demonstrated that miR-101 targeting Rap1A repressed Pca progression.

CRNDE acts as a ceRNA for miR-101 to regulate Rap1A. The ceRNA regulatory network is one main mechanism addressed by lncRNA. To explore whether CRNDE could act as a ceRNA for miR-101, PC3 cells were transfected with si-NC, si-CRNDE, si-CRNDE, and miR-NC inhibitor or miR-101 inhibitor and 22RV1 cells were transfected with pcDNA3.1-NC, pcDNA3.1-CRNDE, pcDNA3.1-CRNDE, and miR-NC mimics or miR-101 mimics. As shown in Figure 7A, the expression of Rap1A protein was significantly decreased by the CRNDE silence, which was attenuated by the miR-101 deficiency in PC3 cells. The data of western blot also revealed that the Rap1A protein level was conspicuously increased by the CRNDE overexpression in 22RV1 cells, while this effect was weakened by the miR-101 addition (Figure 7B). Moreover, as described in Figure 7C, a positive correlation of CRNDE and Rap1A level was displayed in Pca tissues $\left(\mathrm{R}^{2}=0.401, \mathrm{p}<0.0001\right)$.

\section{Discussion}

LncRNAs play a pivotal role in various cellular processes, including proliferation, apoptosis, and metastasis in Pca and are regarded as important targets for diagnosis and treatment of patients [20]. The database of Oncomine suggests a high expression of CRNDE in Pca tissues. Similarly, we also found that the CRNDE expression was elevated in Pca tissues and cells, suggesting that CRNDE might be an oncogenic lncRNA in Pca development. In the present study, we first investigated the cancer-promoting role of CRNDE in Pca cells and showed that it was mediated by miR-101 and Rap1A.

To investigate the effect of CRNDE in Pca progression, the loss- and gain-of-function experiments were performed. In this study, we found that CRNDE promoted cell proliferation by decreasing apoptosis and increasing cell migration and invasion in vitro. The previous study demonstrated that CRNDE could promote tongue squamous cell carcinoma cell growth by regulating the cell cycle process [21]. Whether CRNDE could regulate the cell cycle in Pca cells should be further studied. Moreover, epithelial to mesenchymal transition (EMT) is a key process associated with metastasis and CRNDE could increase cell migration and invasion by inducing EMT $[9,22]$. Hence, we assumed CRNDE regulated the Pca cell migration and invasion by EMT, which should be further validated in the future. The results of this study indicated that CRNDE functioned as an oncogene in Pca.

The lncRNA-mediated ceRNA networks play important roles in Pca progression [23]. To investigate the CRNDEmediated ceRNA network, its target miRNA was explored in Pca cells. The previous studies have indicated miR-217 and miR-384 as important targets of CRNDE in cancer progression $[21,24]$. In the current work, we first confirmed CRNDE as a sponge of miR-101 in Pca by bioinformatics analysis and luciferase reporter assay, suggesting that miR-101 might be required for the CRNDE-mediated progression. Huang et al. reported miR-101 as a tumor suppressor by inhibiting cell viability and inducing apoptosis in Pca cells [25]. Furthermore, Hao et al. suggested that miR-101 repressed Pca cell growth by regulating cyclooxygenase-2 (COX-2) in vitro [26]. Here we also found that miR-101 inhibited cell proliferation, migration, and invasion in Pca. Moreover, the introduction of miR-101 reversed the promoting role of CRNDE in Pca progression, indicating that CRNDE promoted Pca malignancy by sponging miR-101.

To further elucidate the ceRNA network in this research, the target of miR-101 was explored. Multiple mRNAs, such as COX-2, enhancer of zeste homolog 2 (EZH2) and frizzled class receptor 4 (FZD4), have been reported as functional targets of miR-101 [26-28]. This study showed that Rap1A was a key target of miR-101 in Pca. Additionally, the rescue experiments revealed that the therapeutic effect of miR-101 was weakened by the restoration of Rap $1 \mathrm{~A}$, indicating that miR-101 influenced Pca progression by targeting Rap1A. 
A

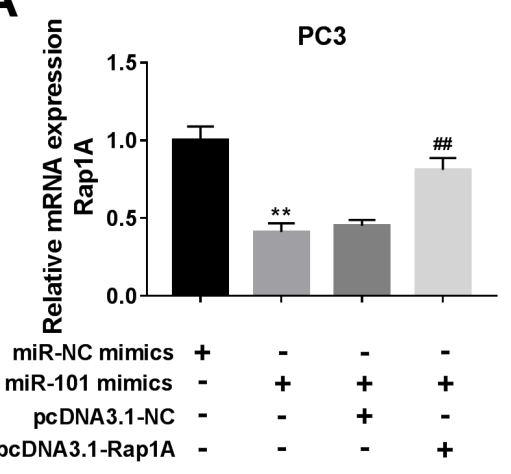

B
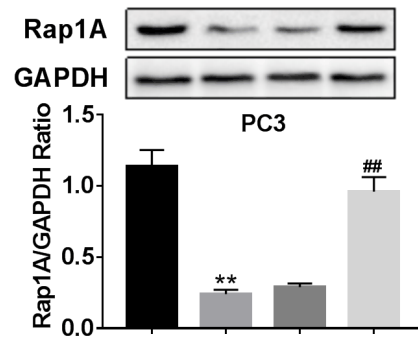

miR-NC mimics + - $\quad-$ miR-101 mimics - $+\quad+\quad+$ pcDNA3.1-NC - $\quad+\quad+\quad-$ PCDNA3.1-Rap1A - $\quad$ - $\quad$ - $\quad+$
C

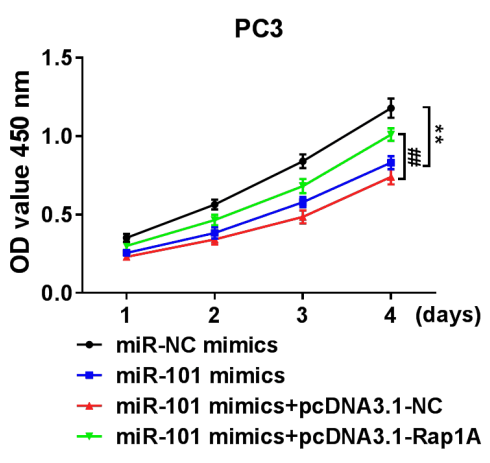

D

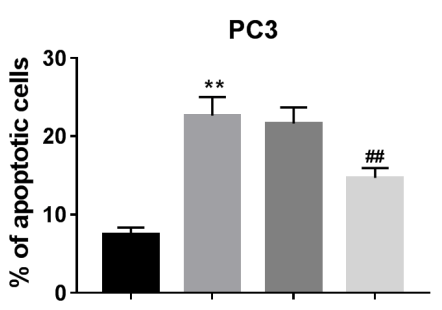

miR-NC mimics + miR-101 mimics -

pcDNA3.1-NC pcDNA3.1-Rap1A -
E

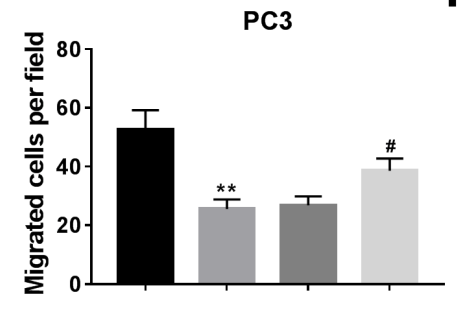

$\mathbf{F}$
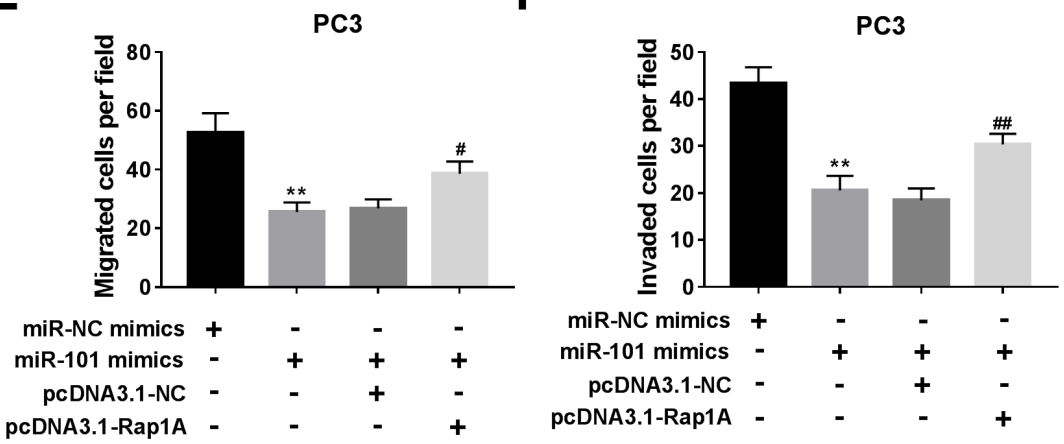
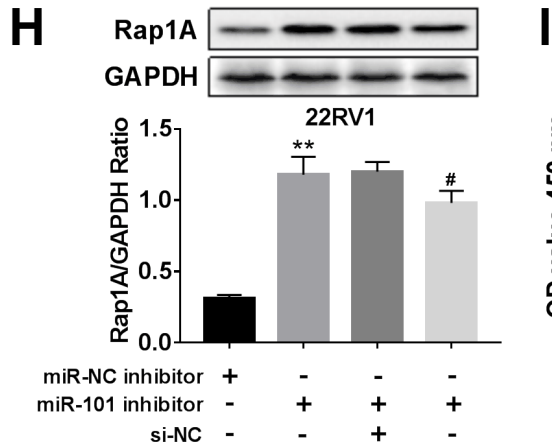

si-NC

si-Rap1A

K

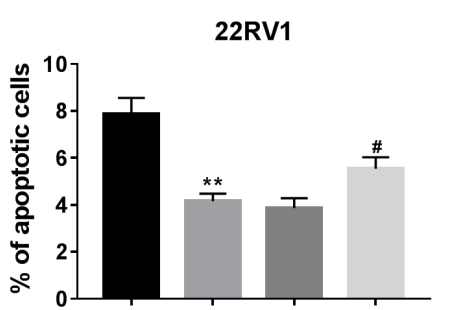

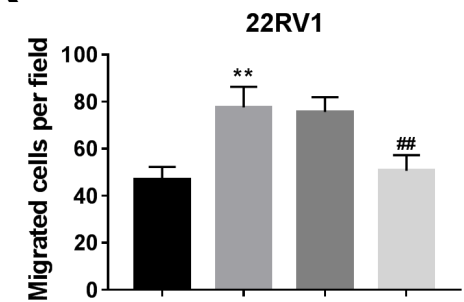

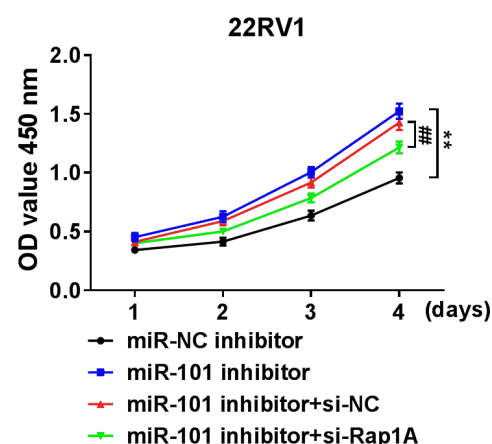

L

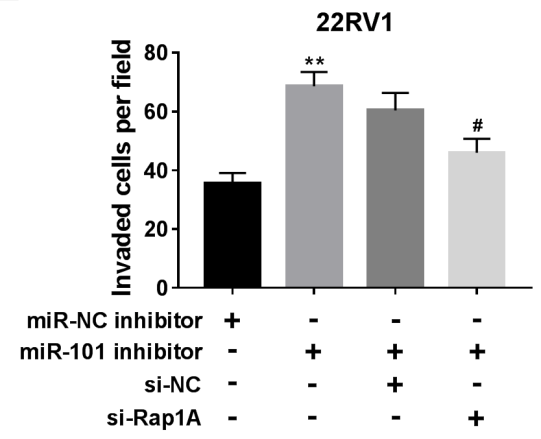

Figure 6. Rap1A reverses the effect of miR-101 on Pca progression. PC3 cells were transfected with miR-NC mimics, miR-101 mimics, miR-101 mimics, and pcDNA3.1-NC or pcDNA3.1-Rap1A. The expression of Rap1A mRNA and protein (A, B), cell proliferation (C), apoptosis (D), migration, and invasion (E, F) were measured in transfected PC3 cells by qRT-PCR, CCK-8, flow cytometry, and transwell assays, respectively. 22RV1 cells were transfected with miR-NC inhibitor, miR-101 inhibitor, miR-101 inhibitor, and si-NC or si-Rap1A. The mRNA and protein expression of miR-101 (G, H), cell proliferation (I), apoptosis $(\mathrm{J})$, migration and invasion $(\mathrm{K}, \mathrm{L})$ were detected in transfected $22 \mathrm{RV} 1$ cells, ${ }^{* *} \mathrm{p}<0.01,{ }^{\#} \mathrm{p}<0.05,{ }^{\# \#} \mathrm{p}<0.01$. 
A
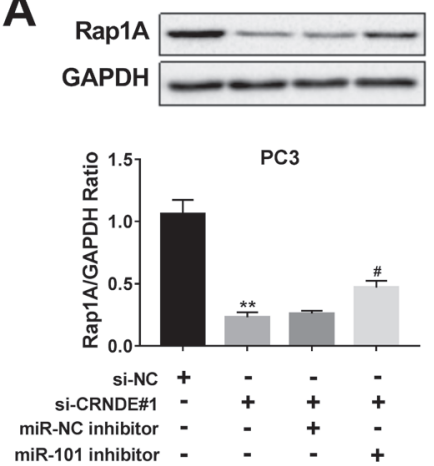

B

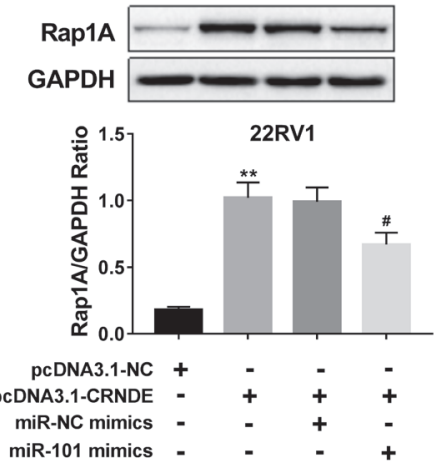

C

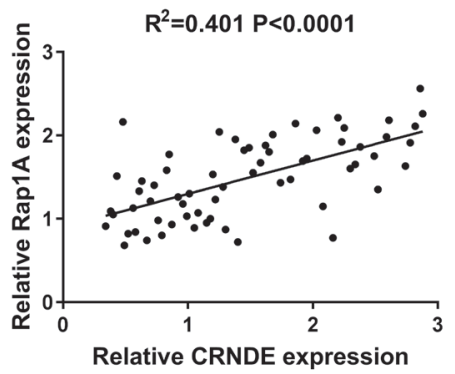

Figure 7. Rap1A expression is regulated by CRNDE and miR-101. A) The expression of Rap1A protein was measured in PC3 cells transfected with si-NC, si-CRNDE, si-CRNDE, and miR-NC inhibitor or miR-101 inhibitor by western blot. B) The level of Rap1 A protein was detected in 22RV1 cells transfected with pcDNA3.1-NC, pcDNA3.1-CRNDE, pcDNA3.1-CRNDE, and miR-101 mimics or miR-NC mimics. C) The association between Rap1A and CRNDE level in Pca tissues was analyzed, ${ }^{* *} \mathrm{p}<0.01,{ }^{*} \mathrm{p}<0.05$.

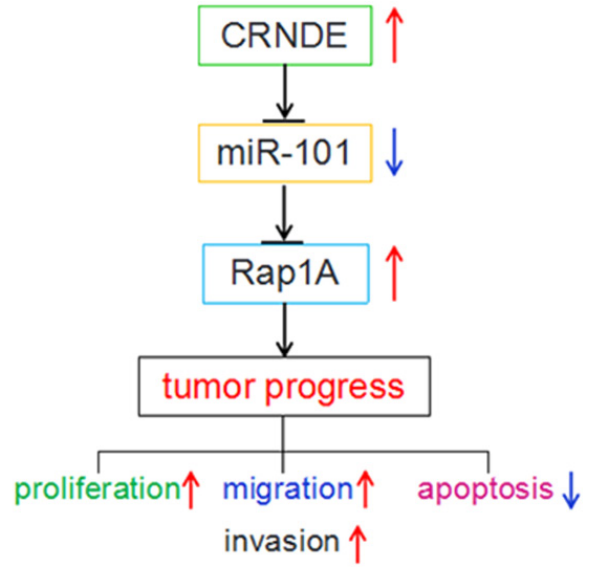

Figure 8. The mechanism mediated by CRNDE in this study. High expression of CRNDE increased Rap1A expression by competitively sponging miR-101, contributing to Pca progression via promoting proliferation, migration, and invasion but inhibiting apoptosis. The up arrow indicated promotion and the down arrow indicated inhibition.

These data also reflected the carcinogenic role of Rap1A, which is also in agreement with the previous study [18]. Besides, by detecting the Rap1A protein level using western blot, miR-101 reduced the expression of Rap1A induced by CRNDE, suggesting that CRNDE might act as a ceRNA for miR-101 to derepress Rap1A expression in Pca cells. However, the in vivo data were absent in the present work. Due to the complex microenvironment in vivo, to better understanding the role of CRNDE in Pca progression, the animal model is expected to be established in a future study.

In conclusion, our data described CRNDE as an oncogenic lncRNA to promote Pca cell proliferation, migration, and invasion, possibly by downregulating miR-101 and upregulating Rap1A (Figure 8). This study indicates that CRNDE could act as a promising target for Pca therapy.

\section{References}

[1] ATTARD G, PARKER C, EELES RA, SCHRODER F, TOMLINS SA et al. Prostate cancer. Lancet 2016; 387: 70-82. https://doi.org/10.1016/S0140-6736(14)61947-4

[2] LITWIN MS, TAN HJ. The diagnosis and treatment of prostate cancer: a review. JAMA 2017; 317: 2532-2542. https:// doi.org/10.1001/jama.2017.7248

[3] KLINGE CM. Non-coding RNAs: long non-coding RNAs and microRNAs in endocrine-related cancers. Endocr Relat Cancer 2018; 25: R259-R282. https://doi.org/10.1530/ERC17-0548

[4] CHAN JJ, TAY Y. Noncoding RNA: RNA regulatory networks in cancer. Int J Mol Sci 2018; 19: E1310. https://doi. org/10.3390/ijms19051310

[5] SUN Y, MA L. New insights into long non-coding RNA MALAT1 in cancer and metastasis. Cancers (Basel) 2019; 11: E216. https://doi.org/10.3390/cancers11020216

[6] XU T, LIN CM, CHENG SQ, MIN J, LI L et al. Pathological bases and clinical impact of long noncoding RNAs in prostate cancer: a new budding star. Mol Cancer 2018; 17: 103. https://doi.org/10.1186/s12943-018-0852-7

[7] DAI J, MU JW, MU H. Long non-coding RNA CRNDE regulates cell proliferation, migration, invasion, epithelialmesenchymal transition and apoptosis in oral squamous cell carcinoma. Oncol Lett 2019; 17: 3330-3340. https://doi. org/10.3892/ol.2019.9978

[8] JING H, XIA H, QIAN M, LV X. Long noncoding RNA CRNDE promotes non-small cell lung cancer progression via sponging microRNA-338-3p. Biomed Pharmacother 2019; 110: 825-833. https://doi.org/10.1016/j.biopha.2018.12.024

[9] ZHU L, YANG N, DU G, LI C, LIU G et al. LncRNA CRNDE promotes the epithelial-mesenchymal transition of hepatocellular carcinoma cells via enhancing the Wnt/beta-catenin signaling pathway. J Cell Biochem 2019; 120: 1156-1164. https://doi.org/10.1002/jcb.26762

[10] MASSILLO C, DALTON GN, FARRE PL, DE LUCA P, DE SIERVI A. Implications of microRNA dysregulation in the development of prostate cancer. Reproduction 2017; 154: R81-R97. https://doi.org/10.1530/REP-17-0322 
[11] WANG CZ, DENG F, LI H, WANG DD, ZHANG W et al. MiR-101: a potential therapeutic target of cancers. Am J Transl Res 2018; 10: 3310-3321.

[12] PANG Y, YOUNG CY, YUAN H. MicroRNAs and prostate cancer. Acta Biochim Biophys Sin (Shanghai) 2010; 42: 363369. https://doi.org/10.1093/abbs/gmq038

[13] GUO J, HUANG X, WANG H, YANG H. Celastrol induces autophagy by targeting AR/miR-101 in prostate cancer cells. PLoS One 2015; 10: e0140745. https://doi.org/10.1371/journal.pone. 0140745

[14] CHAKRAVARTHI BV, GOSWAMI MT, PATHI SS, ROBINSON AD, CIESLIK M et al. MicroRNA-101 regulated transcriptional modulator SUB1 plays a role in prostate cancer. Oncogene 2016; 35: 6330-6340. https://doi.org/10.1038/ onc. 2016.164

[15] CAO XM. Role of miR-337-3p and its target Rap1A in modulating proliferation, invasion, migration and apoptosis of cervical cancer cells. Cancer Biomark 2019; 24: 257-267. https://doi.org/10.3233/CBM-181225

[16] ZHANG T, JIANG K, ZHU X, ZHAO G, WU H et al. miR433 inhibits breast cancer cell growth via the MAPK signaling pathway by targeting Rapla. Int J Biol Sci 2018; 14: 622-632. https://doi.org/10.7150/ijbs.24223

[17] LU L, WANG J, WU Y, WAN P, YANG G. Rap1A promotes ovarian cancer metastasis via activation of ERK/p38 and notch signaling. Cancer Med 2016; 5: 3544-3554. https://doi. org/10.1002/cam4.946

[18] XIANG J, BIAN C, WANG H, HUANG S, WU D. MiR-203 down-regulates Rap1A and suppresses cell proliferation, adhesion and invasion in prostate cancer. J Exp Clin Cancer Res 2015; 34: 8. https://doi.org/10.1186/s13046-015-0125-x

[19] LIVAK KJ, SCHMITTGEN TD. Analysis of relative gene expression data using real-time quantitative PCR and the 2(-Delta Delta C(T)) Method. Methods 2001; 25: 402-408. https://doi.org/10.1006/meth.2001.1262

[20] MITOBE Y, TAKAYAMA KI, HORIE-INOUE K, INOUE S. Prostate cancer-associated lncRNAs. Cancer Lett 2018; 418: 159-166. https://doi.org/10.1016/j.canlet.2018.01.012
[21] REN Y, HE W, CHEN W, MA C, LI Y et al. CRNDE promotes cell tongue squamous cell carcinoma cell growth and invasion through suppressing miR-384. J Cell Biochem 2019; 120: 155-163. https://doi.org/10.1002/jcb.27206

[22] LO UG, LEE CF, LEE MS, HSIEH JT. The role and mechanism of epithelial-to-mesenchymal transition in prostate cancer progression. Int J Mol Sci 2017; 18: E2079. https:// doi.org/10.3390/ijms18102079

[23] YE G, GUO L, XING Y, SUN W, YUAN M. Identification of prognostic biomarkers of prostate cancer with long non-coding RNA-mediated competitive endogenous RNA network. Exp Ther Med 2019; 17: 3035-3040. https://doi.org/10.3892/ etm.2019.7277

[24] WANG H, KE J, GUO Q, BARNABO NAMPOUKIME KP, YANG $P$ et al. Long non-coding RNA CRNDE promotes the proliferation, migration and invasion of hepatocellular carcinoma cells through miR-217/MAPK1 axis. J Cell Mol Med 2018; 22: 5862-5876. https://doi.org/10.1111/jcmm.13856

[25] HUANG S, YANG Z, MA Y, YANG Y, WANG S. miR-101 enhances cisplatin-induced DNA damage through decreasing nicotinamide adenine dinucleotide phosphate levels by directly repressing tp53-induced glycolysis and apoptosis regulator expression in prostate cancer cells. DNA Cell Biol 2017; 36: 303-310. https://doi.org/10.1089/dna.2016.3612

[26] HAO Y, GU X, ZHAO Y, GREENE S, SHA W et al. Enforced expression of miR-101 inhibits prostate cancer cell growth by modulating the COX-2 pathway in vivo. Cancer Prev Res (Phila) 2011; 4: 1073-1083. https://doi.org/10.1158/19406207.CAPR-10-0333

[27] CHEN L, JIA J, ZANG Y, LI J, WAN B. MicroRNA-101 regulates autophagy, proliferation and apoptosis via targeting EZH2 in laryngeal squamous cell carcinoma. Neoplasma 2019; 66: 507-515. https://doi.org/10.4149/ neo_2018_180811N611

[28] CHEN L, LONG Y, HAN Z, YUAN Z, LIU W et al. MicroRNA-101 inhibits cell migration and invasion in bladder cancer via targeting FZD4. Exp Ther Med 2019; 17: 1476-1485. https://doi.org/10.3892/etm.2018.7084 